OPEN ACCESS

Edited by:

Qing-Wu Yang,

Xinqiao Hospital, China

Reviewed by:

Junije Yuan,

Xinqiao Hospital, China

*Correspondence:

Dirk M. Hermann

dirk.hermann@uk-essen.de

Specialty section:

This article was submitted to

Multiple Sclerosis

and Neuroimmunology,

a section of the journal

Frontiers in Immunology

Received: 30 November 2021 Accepted: 21 December 2021

Published: 11 January 2022

Citation:

Mohamud Yusuf A, Hagemann N,

Ludewig P, Gunzer $M$ and

Hermann DM (2022) Roles of

Polymorphonuclear Neutrophils in

Ischemic Brain Injury and Post-

Ischemic Brain Remodeling.

Front. Immunol. 12:825572.

doi: 10.3389/fimmu.2021.825572

\section{Roles of Polymorphonuclear Neutrophils in Ischemic Brain Injury and Post-Ischemic Brain Remodeling}

\author{
Ayan Mohamud Yusuf ${ }^{1,2}$, Nina Hagemann ${ }^{1,2}$, Peter Ludewig ${ }^{3}$, Matthias Gunzer L,5 $^{4,5}$ \\ and Dirk M. Hermann ${ }^{1,2 *}$ \\ ${ }^{1}$ Department of Neurology, University Hospital Essen, Essen, Germany, ${ }^{2}$ Center for Translational and Behavioral \\ Neurosciences, University Hospital Essen, Essen, Germany, ${ }^{3}$ Department of Neurology, University Medical Center Hamburg- \\ Eppendorf, Hamburg, Germany, ${ }^{4}$ Institute of Experimental Immunology and Imaging, University Hospital Essen, \\ Essen, Germany, ${ }^{5}$ Leibniz-Institut für Analytische Wissenschaften-ISAS-e.V., Dortmund, Germany
}

Following ischemic stroke, polymorphonuclear neutrophils (PMNs) are rapidly recruited to the ischemic brain tissue and exacerbate stroke injury by release of reactive oxygen species (ROS), proteases and proinflammatory cytokines. PMNs may aggravate postischemic microvascular injury by obstruction of brain capillaries, contributing to reperfusion deficits in the stroke recovery phase. Thus, experimental studies which specifically depleted PMNs by delivery of anti-Ly6G antibodies or inhibited PMN brain entry, e.g., by CXC chemokine receptor 2 (CXCR2) or very late antigen-4 (VLA-4) blockade in the acute stroke phase consistently reduced neurological deficits and infarct volume. Although elevated PMN responses in peripheral blood are similarly predictive for large infarcts and poor stroke outcome in human stroke patients, randomized controlled clinical studies targeting PMN brain infiltration did not improve stroke outcome or even worsened outcome due to serious complications. More recent studies showed that PMNs have decisive roles in post-ischemic angiogenesis and brain remodeling, most likely by promoting extracellular matrix degradation, thereby amplifying recovery processes in the ischemic brain. In this minireview, recent findings regarding the roles of PMNs in ischemic brain injury and post-ischemic brain remodeling are summarized.

Keywords: focal cerebral ischemia, ischemic stroke, neuroinflammation, brain remodeling, polymorphonuclear neutrophils

\section{BRAIN-INVADING POLYMORPHONUCLEAR NEUTROPHILS (PMNS) AS FIRST LINE IMMUNE RESPONSE CONTRIBUTING TO ISCHEMIC DAMAGE}

PMNs are first line immune invaders in the ischemic brain. PMN recruitment after experimental ischemic stroke is highly coordinated in a spatio-temporal way. PMNs accumulate within capillaries and venules of the ischemic brain territory within the first hour after ischemic stroke (1), followed by their extravasation into the perivascular space and tissue parenchyma, which gets significant in the first two days post-stroke (2-4). Depending on the severity of the ischemic episode, brain PMN 
peak numbers are reached between day 1 and day 3 (5). Transendothelial migration of PMN is mediated by adhesion molecules, e.g., of intercellular adhesion molecule 1 (ICAM-1) and platelet endothelial cell adhesion molecule-1 (PECAM-1), which exhibit an increased expression after induction of experimental ischemic stroke $(6,7)$. Infiltrating PMNs contribute to ischemic brain injury development, as revealed by studies, in which PMNs were depleted by delivery of anti-Ly6G antibodies or in which PMN accumulation was inhibited by anti-very late antigen-4 (VLA4) or anti-CXC chemokine receptor-2 (CXCR2) antibody delivery or mesenchymal stromal cell (MSC)-derived small extracellular vesicle (EV) administration (2, 3, 8). In each of these studies, the prevention of brain PMN brain entry was found to reduce ischemic brain injury and neurological deficits. These studies very clearly defined an injury-exacerbating role of PMNs in the acute stroke phase (9).

\section{PMN HYPERACTIVATION IN PERIPHERAL BLOOD IS ASSOCIATED WITH SECONDARY BRAIN INJURY AND NEUROLOGICAL DETERIORATION}

Evidence in humans supports the idea that PMNs have detrimental consequences on stroke outcome. Hence, PMNs of ischemic stroke patients produce and release significant amounts of reactive oxygen species (ROS) and proteases, such as neutrophil elastase, as shown in peripheral blood within the first 6 hours post-stroke (10). Under ischemic conditions, peripheral blood PMNs are defined by a decreased surface abundance of L-selectin and increased expression of $\beta 2$ integrins (10). This PMN hyperactivation facilitates PMN brain entry and was found to be associated with ischemic stroke progression in human patients $(10,11)$. The loss of microvascular integrity is an important component in this type of secondary brain injury. Thus, increased PMN responses, reflected by increased PMN numbers or, more specifically, a high neutrophil-to-lymphocyte ratio (NLR) in peripheral blood, are associated with an increased likelihood of intracerebral hemorrhage, death and poor neurological outcome at 3 months post-stroke, as assessed by the modified Rankin Scale (mRS) score in patients receiving tissue plasminogen activator (tPA)-induced thrombolysis (12).

\section{MICROVASCULAR OCCLUSION BY PMN STALLS EXACERBATES SECONDARY BRAIN DAMAGE}

A possible mechanism, via which PMNs in peripheral blood aggravate secondary ischemic brain damage are PMN stalls resulting in microvascular occlusions even under conditions of successful arterial reopening (13-15). In a murine thromboembolic stroke model, intravenous thrombolysis initiated 30 minutes after embolization achieved the successful resolution of blood clots in the M2 segment of the middle cerebral artery (14). Yet, as many as $\sim 35 \%$ of capillaries in the evolving infarct core and $\sim 15 \%$ of capillaries in the surrounding infarct periphery exhibited microvascular occlusions compromising brain tissue recovery (14). Strikingly, PMNs made up as many as $67 \%$ of capillary stalls in the infarct core and $54 \%$ of the capillary stalls in the infarct periphery (14). Delivery of anti-Ly6G antibody prior to stroke significantly reduced capillary obstructions and hemorrhagic transformation, and improved tissue perfusion and sensorimotor function after stroke (14).

\section{RANDOMIZED CONTROLLED CLINICAL TRIALS FAILED TO SHOW A BENEFICIAL EFFECT OF PMN INHIBITION STRATEGIES}

Despite numerous studies describing a deleterious role of PMNs post-stroke, randomized controlled clinical trials aiming at reducing PMN brain accumulation did not aid stroke recovery in ischemic stroke patients. Thus, the delivery of a murine monoclonal antibody directed against intercellular adhesion molecule-1 (ICAM1) within 6 hours of symptom onset aggravated neurological recovery assessed by the mRS score, increased stroke mortality and increased infection susceptibility (16). Besides, the administration of the glycoprotein UK279,276, a CD11/CD18 integrin antagonist, within 6 hours of symptom onset did not improve stroke outcome in stroke patients receiving recombinant $\mathrm{tPA}$-induced thrombolysis, as compared to patients receiving tPA alone (17). Functional deficits were also not alleviated after treatment with natalizumab, which is a monoclonal antibody targeting VLA-4 $(18,19)$. Similarly, the delivery of a humanized monoclonal antibody against CD11/ CD18 did not beneficially influence stroke outcome (20). These clinical findings necessitate a more differentiated assessment of the role of PMNs in the ischemic brain. In fact, previous studies largely neglected the post-acute stroke phase in which PMNs have diverse roles.

\section{NEUTROPHIL EXTRACELLULAR TRAPS (NETS) COMPROMISE POST-ISCHEMIC REPERFUSION AND MICROVASCULAR INTEGRITY}

Upon injury, PMNs release NETs, which are composed of PMN DNA, histones and granule components such as elastase and cathepsin G (21). These NETs have recently been reported to impair microvascular integrity after stroke induction in mice by electrocoagulation of the middle cerebral artery (MCA) (22). In this study, NETs were identified based on citrullinated histone H3 (H3Cit) abundance, enrichment of Sytox green-labeled DNA fibers and the presence of the PMN marker Ly6G (22). Disruption of NET formation by means of DNase-1 injection increased blood-brain barrier integrity and increased microvascular survival in the periinfarct cortex (22). The aggravation of microvascular injury by NETs may at least partly be attributed to the impaired lysis of blood clots, 
which abundantly contain citrullinated histones (23). Ex vivo, the treatment of blood clots obtained from ischemic stroke patients with DNAse-1 significantly increased tPA-induced thrombolysis in comparison to tPA alone (23).

\section{N2 POLARIZATION MIGHT CONFER A NEUROPROTECTIVE AND RESTORATIVE PHENOTYPE}

PMNs are a heterogeneous group of leukocytes, which might obtain proinflammatory or anti-inflammatory properties depending on the tissue microenvironment (24). The former PMNs, which are widely referred to as N1 phenotype, contain high levels of proinflammatory cytokines, ROS, Fas (CD95) and ICAM-1 (24-26) (Table 1). These PMNs vigorously recruit and activate $\mathrm{CD}^{+} \mathrm{T}$ cells in tissues (24). On the other hand, so-called N2 PMNs are characterized by their high content of arginase, vascular endothelial growth factor (VEGF), CC-chemokineligand-2 (CCL2) and CC-chemokine-ligand-5 (CCL5) (24, 27, 29) (Table 1). These PMNs may promote tissue survival and remodeling in the ischemic brain (Figure 1). Indeed, treatment with rosiglitazone, an activator of peroxisome proliferatoractivated receptor- $\gamma$ (PPAR $\gamma$ ), increased the number of braininfiltrating PMNs with N2 phenotype at 24 hours after permanent middle cerebral artery occlusion (MCAO) in mice (31). Infarct volume was reduced by rosiglitazone; this neuroprotection was abrogated after PMN depletion induced by anti-PMN antibody delivery (31). In another study, increased numbers of brain-infiltrating PMNs with N2 phenotype were noted in ischemic brains of toll-like receptor-4 deficient compared to wildtype mice after MCAO (32). In this study, PMN accumulation was inversely correlated with infarct volume (32), suggestive of a neuroprotective role of N2 PMNs.

\section{PMNs ARE INDISPENSABLE FOR BRAIN REMODELING IN THE POST-ACUTE STROKE PHASE}

MSC-derived EVs potently promote angiogenesis in the ischemic brain (33), specifically when these EVs are obtained from MSCs

TABLE 1 | Neutrophil polarization.

\begin{tabular}{ll}
\hline Phenotype & Effector molecule \\
\hline N1 neutrophils & ROS (25) \\
& TNF- $\alpha(25)$ \\
& $\operatorname{ICAM}-1(26)$ \\
& Fas (24) \\
\hline N2 neutrophils & $\operatorname{MMPg}(27,28)$ \\
& $\operatorname{VEGF~}(27)$ \\
& Arginase (24) \\
& CCL2, CCL5 (29)
\end{tabular}

Depending on the microenvironment, neutrophils adapt an N1 or N2 phenotype which differ in their effector molecules. Currently, N1 and N2 neutrophils are not distinguishable by distinct surface markers (30). Yet, their biological actions greatly differ from each other, as this table shows. Morphologically, N1 neutrophils are characterized by hypersegmented nuclei (24). cultured under hypoxic conditions $(33,34)$. This effect depends on the presence of PMNs, as recently shown by us. Thus, PMN depletion by anti-Ly6G antibody delivery at 24 hours after MCAO abolished the angiogenic effects of MSC-EVs (34). As an important source of VEGF, PMNs directly stimulate angiogenesis (27). Endothelial cells in turn release growth factors which protect the brain, promote neuroplasticity and induce neurogenesis (35). Newly formed blood vessels support the migration of neuronal progenitor cells from the subventricular zone to the ischemic brain region (36). Inhibition of vascular endothelial growth factor receptor-2 (VEGFR2) impairs the production of growth factors, namely of brain-derived neurotrophic factor (BDNF) (37). Besides, PMNs have important roles in extracellular matrix (ECM) remodeling by production and secretion of matrix metalloproteinase- 9 (MMP9) (28). In rats exposed to MCAO, MMP9 formation was markedly reduced in response to neutropenia induced by the chemotherapeutic vinblastine (38). MMP9 has diverse effects on ischemic injury and brain tissue remodeling, depending on the time-point post-ischemic injury. Post mortem studies in humans revealed robust MMP9 expression in the perivascular space of demarcating brain infarcts (39). PMN-derived MMP9 was localized in proximity to leaky microvessels exhibiting basal lamina disruption (40). Hence, MMP9 expression was found to predispose to hemorrhagic transformation in the acute stroke phase (41). In the post-acute stroke phase, the risk of brain hemorrhage formation has already vanished, and ECM remodeling appears to be a prerequisite for infarct removal and microvascular sprouting (42). In rats exposed to MCAO, delayed MMP inhibition 7 days after MCAO reduced the expression of the neuroplasticity marker early growth response protein-1 (Egr1), decreased the number of RECA- $1^{+}$ endothelial cells, increased sensorimotor deficits and increased ischemic injury, while acute MMP inhibition on day 1 reduced infarct volume (43). In mice, lentiviral MMP9 overexpression initiated on day 7 after MCAO facilitated glial scar resolution, increased microvessel numbers and endogenous neurogenesis and increased the expression of synaptic plasticity markers in the peri-infarct brain (44). The delivery of a vascular endothelial growth factor receptor-2 (VEGFR2) inhibitor or a MMP9 inhibitor reversed these effects (44). Taken together, these results emphasize the close interdependence of postischemic angiogenesis, neurogenesis and synaptic plasticity. All three processes rely on the coordinated reshaping of the ECM.

\section{CONCLUSIONS}

There is robust evidence that PMNs aggravate ischemic brain injury, neurological deficits and hemorrhagic transformation in the acute stroke phase. However, it also becomes clear that PMNs and PMN-derived extracellular matrix proteases are indispensable for successful brain remodeling and neurological recovery in the post-acute stroke phase, perhaps mediated by a restorative N2 phenotype. Future studies will 


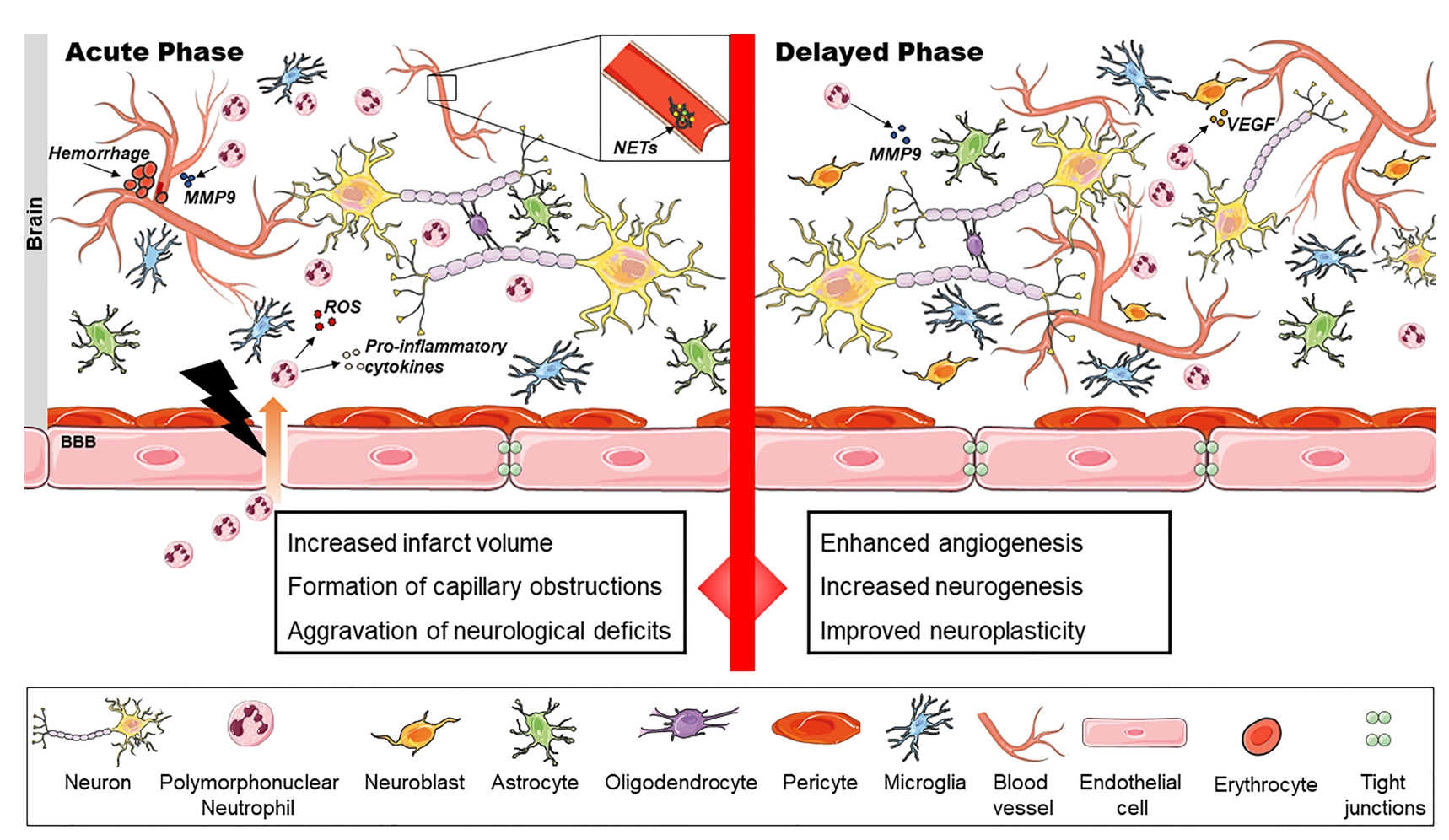

FIGURE 1 | The dual role of polymorphonuclear neutrophils (PMNs) in ischemic stroke. PMNs exacerbate stroke injury in the acute phase by release of reactive oxygen species (ROS) and inflammatory cytokines. Intracerebral hemorrhages may result. Neutrophil extracellular traps (NET) contribute to capillary reperfusion disturbances. In the delayed phase, PMNs enhance cerebral angiogenesis, neurogenesis and neuroplasticity presumably by VEGF release, extracellular matrix (ECM) degradation and remodeling.

have to dissect the diverse roles of PMNs, including proinflammatory $\mathrm{N} 1$ and restorative $\mathrm{N} 2$ phenotypes, define their temporospatial significance and their consequences for ischemic injury and stroke recovery. Only by a thorough understanding of these partly opposing actions, we will be able to define pathophysiological processes via which PMNs can therapeutically be targeted.

\section{AUTHOR CONTRIBUTIONS}

$\mathrm{AM}$ and $\mathrm{DH}$ designed and wrote the article. $\mathrm{NH}, \mathrm{PL}$, and $\mathrm{MG}$ critically revised the article. All authors approved the submitted version.

\section{REFERENCES}

1. Garcia JH, Liu KF, Yoshida Y, Lian J, Chen S, Del Zoppo GJ. Influx of Leukocytes and Platelets in an Evolving Brain Infarct (Wistar Rat). Am J Pathol (1994) 144:188-99.

2. Herz J, Sabellek P, Lane TE, Gunzer M, Hermann DM, Doeppner TR. Role of Neutrophils in Exacerbation of Brain Injury After Focal Cerebral Ischemia in Hyperlipidemic Mice. Stroke (2015) 46:2916-25. doi: 10.1161/ STROKEAHA.115.010620

3. Neumann J, Riek-Burchardt M, Herz J, Doeppner TR, Konig R, Hutten H, et al. Very-Late-Antigen-4 (VLA-4)-Mediated Brain Invasion by Neutrophils

\section{FUNDING}

Supported by the German Research Foundation (FOR-2879 project 405358801 to $\mathrm{DH}, \mathrm{MG}$, and PL and project 389030878 to DH and MG) and Hertie-Stiftung (Hertie Academy of Clinical Neuroscience to PL).

\section{ACKNOWLEDGMENTS}

The authors acknowledge the use of SERVIER Medical Art (https:// smart.servier.com/) for the preparation of the schematic illustration. Servier Medical Art by Servier is licensed under a Creative Commons Attribution 3.0 Unported License.

Leads to Interactions With Microglia, Increased Ischemic Injury and Impaired Behavior in Experimental Stroke. Acta Neuropathol (2015) 129:259-77. doi: 10.1007/s00401-014-1355-2

4. Otxoa-De-Amezaga A, Gallizioli M, Pedragosa J, Justicia C, Miro-Mur F, Salas-Perdomo A, et al. Location of Neutrophils in Different Compartments of the Damaged Mouse Brain After Severe Ischemia/ Reperfusion. Stroke (2019) 50:1548-57. doi: 10.1161/STROKEAHA.118. 023837

5. Gronberg NV, Johansen FF, Kristiansen U, Hasseldam H. Leukocyte Infiltration in Experimental Stroke. J Neuroinflamm (2013) 10:115. doi: 10.1186/1742-2094-10-115 
6. Hagemann N, Mohamud Yusuf A, Martiny C, Zhang X, Kleinschnitz C, Gunzer M, et al. Homozygous Smpd1 Deficiency Aggravates Brain Ischemia/ Reperfusion Injury by Mechanisms Involving Polymorphonuclear Neutrophils, Whereas Heterozygous Smpd1 Deficiency Protects Against Mild Focal Cerebral Ischemia. Basic Res Cardiol (2020) 115:64. doi: 10.1007/s00395-020-00823-x

7. Winneberger J, Schols S, Lessmann K, Randez-Garbayo J, Bauer AT, Mohamud Yusuf A, et al. Platelet Endothelial Cell Adhesion Molecule-1 Is a Gatekeeper of Neutrophil Transendothelial Migration in Ischemic Stroke. Brain Behav Immun (2021) 93:277-87. doi: 10.1016/j.bbi.2020.12.026

8. Wang C, Borger V, Sardari M, Murke F, Skuljec J, Pul R, et al. Mesenchymal Stromal Cell-Derived Small Extracellular Vesicles Induce Ischemic Neuroprotection by Modulating Leukocytes and Specifically Neutrophils. Stroke (2020) 51:1825-34. doi: 10.1161/STROKEAHA.119.028012

9. Hermann DM, Gunzer M. Polymorphonuclear Neutrophils Play a Decisive Role for Brain Injury and Neurological Recovery Poststroke. Stroke (2019) 50: e40-1. doi: 10.1161/STROKEAHA.118.021564

10. Weisenburger-Lile D, Dong Y, Yger M, Weisenburger G, Polara GF, Chaigneau T, et al. Harmful Neutrophil Subsets in Patients With Ischemic Stroke: Association With Disease Severity. Neurol Neuroimmunol Neuroinflamm (2019) 6:e571. doi: 10.1212/NXI.0000000000000571

11. Hermann DM, Gunzer M. Contribution of Polymorphonuclear Neutrophils in the Blood Periphery to Ischemic Brain Injury. Neurol Neuroimmunol Neuroinflamm (2019) 6:e570. doi: 10.1212/NXI.00000000000000570

12. Maestrini I, Strbian D, Gautier S, Haapaniemi E, Moulin S, Sairanen T, et al. Higher Neutrophil Counts Before Thrombolysis for Cerebral Ischemia Predict Worse Outcomes. Neurology (2015) 85:1408-16. doi: 10.1212/WNL.0000000000002029

13. Del Zoppo GJ, Mabuchi T. Cerebral Microvessel Responses to Focal Ischemia. J Cereb Blood Flow Metab (2003) 23:879-94. doi: 10.1097/01.WCB. 0000078322.96027 .78

14. El Amki M, Gluck C, Binder N, Middleham W, Wyss MT, Weiss T, et al. Neutrophils Obstructing Brain Capillaries Are a Major Cause of No-Reflow in Ischemic Stroke. Cell Rep (2020) 33:108260. doi: 10.1016/j.celrep.2020.108260

15. Rolfes L, Riek-Burchardt M, Pawlitzki M, Minnerup J, Bock S, Schmidt M, et al. Neutrophil Granulocytes Promote Flow Stagnation Due to Dynamic Capillary Stalls Following Experimental Stroke. Brain Behav Immun (2021) 93:322-30. doi: 10.1016/j.bbi.2021.01.011

16. Enlimomab Acute Stroke Trial Investigators. Use of Anti-ICAM-1 Therapy in Ischemic Stroke: Results of the Enlimomab Acute Stroke Trial. Neurology (2001) 57:1428-34. doi: 10.1212/WNL.57.8.1428

17. Krams M, Lees KR, Hacke W, Grieve AP, Orgogozo JM, Ford GA, et al. Acute Stroke Therapy by Inhibition of Neutrophils (ASTIN): An Adaptive DoseResponse Study of UK-279,276 in Acute Ischemic Stroke. Stroke (2003) 34:2543-8. doi: 10.1161/01.STR.0000092527.33910.89

18. Elkins J, Veltkamp R, Montaner J, Johnston SC, Singhal AB, Becker K, et al. Safety and Efficacy of Natalizumab in Patients With Acute Ischaemic Stroke (ACTION): A Randomised, Placebo-Controlled, Double-Blind Phase 2 Trial. Lancet Neurol (2017) 16:217-26. doi: 10.1016/S1474-4422(16)30357-X

19. Elkind MSV, Veltkamp R, Montaner J, Johnston SC, Singhal AB, Becker K, et al. Natalizumab in Acute Ischemic Stroke (ACTION II): A Randomized, Placebo-Controlled Trial. Neurology (2020) 95:e1091-104. doi: 10.1212/ WNL.0000000000010038

20. Becker KJ. Anti-Leukocyte Antibodies: LeukArrest (Hu23F2G) and Enlimomab (R6.5) in Acute Stroke. Curr Med Res Opin (2002) 18(Suppl 2): s18-22. doi: 10.1185/030079902125000688

21. Brinkmann V, Reichard U, Goosmann C, Fauler B, Uhlemann Y, Weiss DS, et al. Neutrophil Extracellular Traps Kill Bacteria. Science (2004) 303:1532-5. doi: 10.1126/science.1092385

22. Kang L, Yu H, Yang X, Zhu Y, Bai X, Wang R, et al. Neutrophil Extracellular Traps Released by Neutrophils Impair Revascularization and Vascular Remodeling After Stroke. Nat Commun (2020) 11:2488. doi: 10.1038/ s41467-020-16191-y

23. Laridan E, Denorme F, Desender L, Francois O, Andersson T, Deckmyn H, et al. Neutrophil Extracellular Traps in Ischemic Stroke Thrombi. Ann Neurol (2017) 82:223-32. doi: 10.1002/ana.24993

24. Fridlender ZG, Sun J, Kim S, Kapoor V, Cheng G, Ling L, et al. Polarization of Tumor-Associated Neutrophil Phenotype by TGF-Beta: "N1" Versus "N2" TAN. Cancer Cell (2009) 16:183-94. doi: 10.1016/j.ccr.2009.06.017
25. Mishalian I, Bayuh R, Levy L, Zolotarov L, Michaeli J, Fridlender ZG. TumorAssociated Neutrophils (TAN) Develop Pro-Tumorigenic Properties During Tumor Progression. Cancer Immunol Immunother (2013) 62:1745-56. doi: 10.1007/s00262-013-1476-9

26. Andzinski L, Kasnitz N, Stahnke S, Wu CF, Gereke M, Von KockritzBlickwede M, et al. Type I IFNs Induce Anti-Tumor Polarization of Tumor Associated Neutrophils in Mice and Human. Int J Cancer (2016) 138:1982-93. doi: $10.1002 / \mathrm{ijc} .29945$

27. Jablonska J, Leschner S, Westphal K, Lienenklaus S, Weiss S. Neutrophils Responsive to Endogenous IFN-Beta Regulate Tumor Angiogenesis and Growth in a Mouse Tumor Model. J Clin Invest (2010) 120:1151-64. doi: 10.1172/JCI37223

28. Zou JM, Qin J, Li YC, Wang Y, Li D, Shu Y, et al. IL-35 Induces N2 Phenotype of Neutrophils to Promote Tumor Growth. Oncotarget (2017) 8:33501-14. doi: 10.18632/oncotarget.16819

29. Shaul ME, Levy L, Sun J, Mishalian I, Singhal S, Kapoor V, et al. TumorAssociated Neutrophils Display a Distinct N1 Profile Following TGFbeta Modulation: A Transcriptomics Analysis of Pro- vs. Antitumor TANs. Oncoimmunology (2016) 5:e1232221. doi: 10.1080/2162402X.2016.1232221

30. Ohms M, Moller S, Laskay T. An Attempt to Polarize Human Neutrophils Toward N1 and N2 Phenotypes. vitro Front Immunol (2020) 11:532. doi: 10.3389/fimmu.2020.00532

31. Cuartero MI, Ballesteros I, Moraga A, Nombela F, Vivancos J, Hamilton JA, et al. N2 Neutrophils, Novel Players in Brain Inflammation After Stroke: Modulation by the PPARgamma Agonist Rosiglitazone. Stroke (2013) 44:3498-508. doi: 10.1161/STROKEAHA.113.002470

32. Garcia-Culebras A, Duran-Laforet V, Pena-Martinez C, Moraga A, Ballesteros I, Cuartero MI, et al. Role of TLR4 (Toll-Like Receptor 4) in N1/N2 Neutrophil Programming After Stroke. Stroke (2019) 50:2922-32. doi: 10.1161/STROKEAHA.119.025085

33. Doeppner TR, Herz J, Gorgens A, Schlechter J, Ludwig AK, Radtke S, et al. Extracellular Vesicles Improve Post-Stroke Neuroregeneration and Prevent Postischemic Immunosuppression. Stem Cells Transl Med (2015) 4:1131-43. doi: $10.5966 / \mathrm{sctm} .2015-0078$

34. Gregorius J, Wang C, Stambouli O, Hussner T, Qi Y, Tertel T, et al. Small Extracellular Vesicles Obtained From Hypoxic Mesenchymal Stromal Cells Have Unique Characteristics That Promote Cerebral Angiogenesis, Brain Remodeling and Neurological Recovery After Focal Cerebral Ischemia in Mice. Basic Res Cardiol (2021) 116:40. doi: 10.1007/s00395-021-00881-9

35. Shen Q, Goderie SK, Jin L, Karanth N, Sun Y, Abramova N, et al. Endothelial Cells Stimulate Self-Renewal and Expand Neurogenesis of Neural Stem Cells. Science (2004) 304:1338-40. doi: 10.1126/science.1095505

36. Kojima T, Hirota Y, Ema M, Takahashi S, Miyoshi I, Okano H, et al. Subventricular Zone-Derived Neural Progenitor Cells Migrate Along a Blood Vessel Scaffold Toward the Post-Stroke Striatum. Stem Cells (2010) 28:545-54. doi: 10.1002/stem.306

37. Chen J, Zhang C, Jiang H, Li Y, Zhang L, Robin A, et al. Atorvastatin Induction of VEGF and BDNF Promotes Brain Plasticity After Stroke in Mice. J Cereb Blood Flow Metab (2005) 25:281-90. doi: 10.1038/sj.jcbfm.9600034

38. Justicia C, Panes J, Sole S, Cervera A, Deulofeu R, Chamorro A, et al. Neutrophil Infiltration Increases Matrix Metalloproteinase-9 in the Ischemic Brain After Occlusion/Reperfusion of the Middle Cerebral Artery in Rats. J Cereb Blood Flow Metab (2003) 23:1430-40. doi: 10.1097/ 01.WCB.0000090680.07515.C8

39. Rosell A, Ortega-Aznar A, Alvarez-Sabin J, Fernandez-Cadenas I, Ribo M, Molina CA, et al. Increased Brain Expression of Matrix Metalloproteinase-9 After Ischemic and Hemorrhagic Human Stroke. Stroke (2006) 37:1399-406. doi: 10.1161/01.STR.0000223001.06264.af

40. Rosell A, Cuadrado E, Ortega-Aznar A, Hernandez-Guillamon M, Lo EH, Montaner J. MMP-9-Positive Neutrophil Infiltration is Associated to BloodBrain Barrier Breakdown and Basal Lamina Type IV Collagen Degradation During Hemorrhagic Transformation After Human Ischemic Stroke. Stroke (2008) 39:1121-6. doi: 10.1161/STROKEAHA.107.500868

41. Montaner J, Molina CA, Monasterio J, Abilleira S, Arenillas JF, Ribo M, et al. Matrix Metalloproteinase-9 Pretreatment Level Predicts Intracranial Hemorrhagic Complications After Thrombolysis in Human Stroke. Circulation (2003) 107:598-603. doi: 10.1161/01.CIR.0000046451.38849.90

42. Edgar LT, Underwood CJ, Guilkey JE, Hoying JB, Weiss JA. Extracellular Matrix Density Regulates the Rate of Neovessel Growth and Branching in 
Sprouting Angiogenesis. PloS One (2014) 9:e85178. doi: 10.1371/ journal.pone.0085178

43. Zhao BQ, Wang S, Kim HY, Storrie H, Rosen BR, Mooney DJ, et al. Role of Matrix Metalloproteinases in Delayed Cortical Responses After Stroke. Nat Med (2006) 12:441-5. doi: 10.1038/nm1387

44. Cai H, Ma Y, Jiang L, Mu Z, Jiang Z, Chen X, et al. Hypoxia Response Element-Regulated MMP-9 Promotes Neurological Recovery via Glial Scar Degradation and Angiogenesis in Delayed Stroke. Mol Ther (2017) 25:144859. doi: 10.1016/j.ymthe.2017.03.020

Conflict of Interest: The authors declare that the research was conducted in the absence of any commercial or financial relationships that could be construed as a potential conflict of interest.
Publisher's Note: All claims expressed in this article are solely those of the authors and do not necessarily represent those of their affiliated organizations, or those of the publisher, the editors and the reviewers. Any product that may be evaluated in this article, or claim that may be made by its manufacturer, is not guaranteed or endorsed by the publisher.

Copyright (c) 2022 Mohamud Yusuf, Hagemann, Ludewig, Gunzer and Hermann. This is an open-access article distributed under the terms of the Creative Commons Attribution License (CC BY). The use, distribution or reproduction in other forums is permitted, provided the original author(s) and the copyright owner(s) are credited and that the original publication in this journal is cited, in accordance with accepted academic practice. No use, distribution or reproduction is permitted which does not comply with these terms. 